\title{
Implicit Associations in Blood-Injection-Injury Phobia: Changes after Exposure to a Disgust-Eliciting Stimulus
}

\author{
Lauren A. Rothstein, Alanna M Covington and Michele M Carter \\ American University, Department of Psychology, 321 Asbury Bldg, 4400 Massachusetts Avenue NW, Washington, DC 20016, USA
}

\begin{abstract}
Objective: The current study aimed to examine implicit disgust associations between blood injection-injury (BII) phobic and non-phobic individuals.

Method: The implicit disgust associations between 30 blood-injection-injury (BII) phobic 30 and non-phobic individuals were evaluated following 30-minute in vivo exposure to a disgust eliciting stimulus (severed deer leg). Participants engaged in an implicit association test (IAT), prior to and following exposure to determine the strength of implicit associations regarding the concept of disgust. Participants also engaged in a behavior approach/avoidance task (BAT) with a vial of blood at the same time points to determine if disgust elicited by the deer leg was generalizable to a BII-specific disgust-elicitor. IAT and BAT assessments were then repeated one week following exposure.
\end{abstract}

Results: A significant change in implicit associations was found from pre-exposure to follow-up. There was also a significant decrease in avoidance to the BAT from pre-exposure to follow-up.

Conclusions: BII phobic individuals underwent a cognitive change and lessening of disgust associations.

Keywords: Disgust; Phobias; Blood-injection-injury; Implicit associations; Exposure

\section{Introduction}

Blood-injection-injury (BII) phobia is a subtype of specific phobia characterized by anxiety or fear prompted by encountering blood or injuries, by seeing or having to receive an injection, or any other type of invasive medical procedure [1]. Since the fear or anxiety typically leads to avoidance behaviors, an individual with BII phobia may avoid potentially lifesaving or routine medical procedures, which can be a detriment to physical health and well being [2]. As avoiders of medical settings, individuals with BII phobia are less likely to present for treatment of their specific phobia [1].

Cognitive models posit that schemas, or mental structures, that represent particular objects or characteristics in the world, influence the processing of information [3]. Faulty information processing, or a maladaptive schema, can lead to psychopathology by making individuals more attuned to threatening cues, more likely to construe vague cues as threatening, and more likely to recollect cues that are pertinent to their particular cognitive fear schema [3-5].

Individuals with a specific phobia are likely to have cognitions containing maladaptive beliefs that focus on physical or psychological threats resulting from encounters with the feared stimuli despite the fact that these beliefs are not necessarily accurate or supported by evidence from experience. Such beliefs serve to constantly reinforce anxiety, and symptoms cannot be easily alleviated without a change in the fear schema $[4,6]$. Although the fear schema was traditionally the most commonly discussed schema in cognitive conceptualizations of specific phobias, recent research suggests that disgust plays a more significant role in the acquisition and maintenance of BII phobia [79]. Currently, there are three types of disgust most examined in the literature: animal reminder, core, and contamination. Animal reminder disgust is the type most commonly examined in relation to BII phobia $[10,11]$.

The role of disgust in BII phobia has been assessed through a variety of methods including self-report [11-13], behavioral tasks [10,12-14], and implicit association tests [15]. Empirically, individuals categorized as BII phobic are consistently elevated on disgust sensitivity measures compared to their non-phobic counterparts [11,12]. Furthermore [14], found that higher levels of self reported disgust in BII phobics led to increased avoidance on a behavior approach/avoidance task (BAT) with an animal reminder disgust elicitor and a core disgust elicitor [7] found high BII fear to be associated with increased behavioral avoidance of an animal reminder stimulus.

Consequently, several studies have addressed the construct of disgust in treatment [16] examined the efficacy of a one-session treatment for specific phobia among a subclinical population. While they found participants demonstrated less behavioral avoidance and a decline in global disgust sensitivity, they did not offer a condition that exposed participants to a primarily disgust-inducing stimulus and therefore were not able to address the role of disgust separate from fear. Furthermore, there was no attempt to assess how exposure may or may not alter the cognitive processing postulated by cognitive theorists to be central in specific phobia symptoms.

Additionally, researchers have evaluated cognitive processes of specific phobias utilizing an Implicit Association Test (IAT). IATs have been used to measure the degree of association between two concepts or ideas in an individual's cognitive network. The IAT is a computer based matching task that requires the participant to sort images

*Corresponding author: Carter M, American University, Department of Psychology, 321 Asbury Bidg, 4400 Massachusetts Avenue NW, Washington, DC 20016, USA, Tel: (202) 885-1712; Fax: 202-885-1023; E-mail: mcart@american. edu

Received: November 04, 2015; Accepted: November 06, 2015; Published: November 30, 2015

Citation: Rothstein LA, Covington AM, Carter MM (2015) Implicit Associations in Blood-Injection-Injury Phobia: Changes after Exposure to a Disgust-Eliciting Stimulus. Clin Exp Psychol 1: 105. doi:10.4172/cep.1000105

Copyright: (c) 2015 Rothstein LA, et al. This is an open-access article distributed under the terms of the Creative Commons Attribution License, which permits unrestricted use, distribution, and reproduction in any medium, provided the original author and source are credited. 
and words into two corresponding paired superordinate categories (e.g. disgusting or appealing, and mutilation or flowers). The primary underlying assumption of the IAT is that processing speed (response latency) indirectly measures the strength of these connections and consequently the strength of the individual's related cognitive schemas The primary underlying assumption in the use of IATs to study schemas is that processing speed (response latency) indirectly measures the degree of association between two concepts or ideas in the cognitive network, or schema, of an individual [5].

Teachman et al. [5] utilized an IAT to examine the cognitive associations of individuals with snake phobia and spider phobia and found that participants with either phobia responded with faster reaction times to pairings of words that are relevant to their particular specific phobia.

In a later study [6], provided spider phobic individuals with three 90-minute cognitive-behavioral therapy (CBT) group treatment sessions. Participants were required to complete four IATs (i.e., goodbad, danger-safety, disgusting-appealing, afraid unafraid) both before and after group treatment. Results showed that reaction times between the stimulus of interest (spider) and disgusting and afraid slowed, indicating that the maladaptive associations between spider and disgusting/afraid reduced post-group therapy.

The current study sought to identify whether or not exposure to a disgust-eliciting stimulus would reduce maladaptive associations among BII phobic individuals, as evidenced by a change in reaction time on disgust-mutilation pairings from the IAT following exposure. It was predicted that after undergoing 30 minutes of exposure to an animal reminder disgust elicitor (severed deer leg; as used in) [7], participants categorized as BII phobic would have significantly slower reaction times on the disgust IAT than they did at baseline. Additionally, slower response times would be maintained at follow-up, indicating participants did not return to baseline response times. Moreover, the further a BII phobic participant progresses through the exposure hierarchy, the greater cognitive change they were expected to exhibit.

Prior research has demonstrated the efficacy of exposure to phobia-relevant stimuli to decrease phobic avoidance. Participants in the current study were assessed using a BAT with a phobia-relevant disgust-eliciting stimulus (vial of blood) to determine whether or not exposure to the severed deer leg was generalizable to another animal reminder disgust-elicitor. It was predicted that BII phobic participants would demonstrate a reduction in avoidance behavior after undergoing 30 minutes of exposure to the severed deer leg. It was hypothesized that at follow-up decreases in avoidance would be maintained. In addition, it was predicted that NP individuals would be able to complete the BAT in its entirety at all assessment points, as they should not exhibit avoidance behaviors.

\section{Method}

\section{Participants}

Sixty individuals (30 BII phobic; 30 non-phobic) from a mediumsized university in the mid-Atlantic region of the United States participated in the current study. The majority of participants selfidentified as Caucasian (71.7\%), female (86.7\%), and had a mean age of 19.6 years old. Phobic participants were required to meet DSM-IV criteria for BII phobia and demonstrate avoidance in the initial BAT. Any participants who were currently in or had previously received treatment for BII phobia were excluded from this study. No participants were excluded on this basis.

\section{Measures}

\section{Demographic Questionnaires}

The demographics questionnaire assessed participant sex, age, ethnicity, previous participation in a similar study, and treatment history.

\section{Anxiety Disorders Interview Schedule for DSM-IV (ADIS- IV;[17])}

The specific phobia section of the ADIS-IV was administered by trained interviewers. Diagnoses were rated on an 8-point severity scale with scores of four or greater indicating the presence of a clinical diagnosis.

\section{Disgust Scale-Revised (DS-R; [13,18])}

The DS- $\mathrm{R}$ is a 25 -item trait questionnaire developed to measure disgust sensitivity. Items in the questionnaire represent three distinct categories (subscales) of disgust: core disgust, animal reminder disgust, and contamination disgust $[8,18]$. Cronbach's alpha for the overall scale is reported to be good $(\alpha=.81)$. Similarly, Cronbach's alpha for the core, $(\alpha=.80)$, animal reminder $(\alpha=.82)$, and contamination subscales $(\alpha=$ .71) are good. In the current study, internal consistency reliability for the DS-R overall was good $(\alpha=.87)$. Internal consistency reliability for the core, animal reminder, and contamination subscales were .74, .79, and .70 , respectively.

\section{Beck Depression Inventory-Second Edition (BDI-II; [19])}

The BDI-II contains 21 items and measures affective and somatic symptoms of depression per [6], participants scoring in the moderate to severe range for depressive symptoms (score above 21 ) were excluded due to the potential influence on reaction time. When examined in a population of college students, the BDI-II demonstrated good internal consistency reliability (Cronbach's alpha $=.90 ;[20]$ ). In the current study, internal consistency reliability for the BDI-II was $\alpha=.80$.

\section{Implicit Association Test [21]}

An IAT is a timed response-task in which participants classify words or images into superordinate categories. Response times for classification are recorded by a computer program and are said to measure the association between two concepts in an individual's cognitive network. Smaller reaction times indicate closer associations between concepts (See https://implicit.harvard.edu/implicit/ demo/ for a sample test and information about IATs).

In accordance with part of the procedure employed by [5], one set of opposing descriptive categories was used for the IAT: disgustingappealing. The current study utilized mutilation images from [22], which portray a variety of bloody injuries to arms, legs, feet, and hands; the images contain only the injured body part. The neutral stimuli (flowers) were selected from the International Affective Picture System (IAPS; [23]). For each of the two categories (mutilation and flowers), ten pictures were utilized, producing 20 total images in the IAT. Word stimuli, borrowed from [5], were also used in the IAT.

Response latencies in milliseconds were recorded for the disgustingmutilation IAT using the DirectRT software program before and after the participant underwent 30 minutes of in vivo exposure to an animal reminder disgust-eliciting stimulus. Therefore, one measure of interest was the difference between response latencies for each participant before and after exposure. In order to examine the difference, two primary reaction times, one for the disgust IAT that was completed 
pre-exposure to the severed deer leg and one completed post-exposure, were calculated for each participant upon completion of the disgust IAT.

These times were then converted to D scores, which correct for the confounding variable of general cognitive ability [24]. D scores were calculated according to the method proposed by [24]. Each D score falls between -2 and +2 . The more positive the $\mathrm{D}$ score, the more the person believes that mutilation images are most closely paired with the concept of disgusting (rather than appealing).

\section{Exposure}

Participants underwent 30 minutes of in vivo exposure to a severed deer leg [25]. The exposure hierarchy consisted of five total steps: Step 1. Looking at the deer leg that will be placed on the table in front of the participant; Step 2. Touching a spot on the table right next to the deer leg while wearing a latex glove; Step 3 . Touching a spot on the table right next to the deer leg while wearing no glove; Step 4 . Touching the deer leg (the bloody part) while wearing a glove; and, Step 5. Pressing a gloveless finger against the bloody part of the deer leg (participants were only required to respond "yes" or "no" to the final step and were not instructed to engage in the step, even if they agreed). Upon completing each step, participants were asked to report their subjective level of disgust verbally on a scale from $0-10$ (0 representing no disgust experienced and 10 indicating the most disgust). Participants that indicated they were willing to engage in a step were only prompted to move on to the next step in the hierarchy once they reported that their subjective disgust rating was 4 or less on the $0-10$ scale. Declining to participate in any step in the hierarchy meant that their subjective disgust rating must be zero before they were asked again about their willingness to engage in the next step. Exposure to the severed deer leg was terminated if all 5 steps of the exposure hierarchy were completed, the 30- minute time limit had been met, or a participant chose to withdraw from the study. All BII phobic individuals, with the exception of two participants, completed the full 30 minutes of exposure to the severed deer leg. In the two incidences in which participants indicated that they no longer wished to participate in the exposure and/or study, the deer leg was removed from their presence and their participation concluded. The data for the two BII participants that withdrew was not included in any analyses. Both subjective disgust ratings and highest step completed in the exposure hierarchy were noted for all participants by researcher.

\section{Behavioral Approach/Avoidance Task (BAT)}

Participants engaged in a pre- and post-exposure BAT. The BAT consisted of several tasks that a participant was asked to complete with another disgust-eliciting stimulus (vial of fake blood). If the participant declined to engage in a step, the BAT was discontinued and the step at which the participant declined engagement was noted.

\section{Procedure}

After providing informed consent, participants completed the packet of questionnaires followed by administration of the ADIS. Volunteers then engaged in the pre-exposure BAT with a vial of blood followed by the pre-exposure disgust IAT. Next, participants completed the 30-minute exposure to the severed deer leg, the post-exposure disgust IAT, and the post-exposure BAT. Participants returned 6-9 days later to complete the DS-R, a BAT, and the IAT. The same stimuli were used for the follow-up session as the initial session. Upon completion of the follow up portion, participants were debriefed and compensated.

\section{Results}

\section{Demographic Information}

No significant differences emerged between BII phobic and nonphobic individuals in terms of mean age, gender distribution, race/ ethnicity, and/or mean BDI-II scores (Table 1).

\section{Disgust Scale Results}

The overall mean for the DS- $\mathrm{R}$ in random samples of university undergraduate students is comparable to those participants categorized as non-phobic in the current study ( $M=51.86$ in [26]; $M=50.45$ in [27]. There was a significant difference in mean total scores on the Disgust Scale-Revised between BII phobic and non-phobic individuals, $t(58)$ $=-5.02, \mathrm{p}<.001$, with BII phobic individuals evidencing significantly higher mean DS-R scores than non-phobic participants (Table 1). Independent samples t-tests were also conducted for each subscale of the DS-R to determine whether or not there was a difference in means between BII phobic and non-phobic individuals. There was a significant difference between BII phobic and non-phobic individuals on the animal reminder disgust, $(t(58)=-6.41, p<.001)$, core disgust subscales $(t(58)=-3.52, p=.001)$, and contamination subscales $(t(58)$ $=2.10, \mathrm{p}=.04)$.

Hypothesis 1: The first hypothesis predicted that after undergoing 30 minutes of exposure to an animal reminder disgust elicitor (severed deer leg), participants categorized as BII phobic would have significantly slower reaction times on the disgust IAT than they did at baseline. A paired samples t-test was conducted to test this hypothesis, and the hypothesis was partially supported. Although there was no significant difference for BII phobic individuals in $\mathrm{D}$ scores between preexposure and post-exposure, $t(29)=1.57, p=.13$, significant differences were found from pre exposure to follow-up, $t(29)=3.13, p=.004$. Nonphobic individuals evidenced no significant difference in D scores between pre- and post-exposure, $t(29)=1.16, p=.26$, or pre-exposure to follow-up, $\mathrm{t}(29)=1.92, \mathrm{p}=.065$ (see Table 2).

Hypothesis 2: The second hypothesis predicted that the further a BII phobic participant progressed through the exposure hierarchy with the severed deer leg, the greater the difference in $\mathrm{D}$ scores between preand post-exposure $(\triangle \mathrm{D} 1)$ and between pre-exposure and follow-up $(\Delta \mathrm{D} 2)$ the participant would exhibit. Two difference in D scores were calculated as follows: 1$)$ preexposure $\mathrm{D}$ score - post-exposure $\mathrm{D}$ score $=(\Delta \mathrm{D} 1)$ and 2) pre-exposure $\mathrm{D}$ score - follow-up $\mathrm{D}$ score $=(\Delta \mathrm{D} 2)$. If the resulting values are positive, it indicates a lessening of strength of associations between mutilation-disgusting. To test this hypothesis, correlations were conducted to examine relationships between the progression through the hierarchy (a score from 0 to 5), and difference scores. The hypothesis was not supported, as there was not a significant correlation between progression through the hierarchy and $\Delta \mathrm{D} 1$ score, $r=.19, p=.14$ or $\Delta \mathrm{D} 2$ score, $r=-.10, \mathrm{p}=.44$.

Of BII phobic individuals during the exposure to the severed deer leg, $43.3 \%$ were not willing to look at the leg, $3.3 \%$ were willing to look at the deer leg but do nothing more, $3.3 \%$ were willing to touch a spot next to the leg on the table with a gloved hand but nothing more, $10.0 \%$ were willing to touch a spot on the table next to the leg with no glove on but nothing more, $20 \%$ were willing to touch the leg with a glove on but nothing more, and $20 \%$ completed the BAT in its entirety by agreeing to touch the deer leg with a gloveless hand (though they were not instructed to do so). All of the NP individuals were willing to look at the leg and touch a spot next to the leg on the table with a gloved hand, $96.7 \%$ were willing to touch the leg with a glove on, $86.7 \%$ were 


\begin{tabular}{|c|c|c|c|c|}
\hline \multirow[b]{3}{*}{ Gender } & \multicolumn{4}{|c|}{ Participant Information by Group } \\
\hline & \multirow[t]{2}{*}{ Bll phobic } & \multicolumn{2}{|c|}{ Non-phobic } & \\
\hline & & & & \\
\hline Female & 27 & 25 & & \\
\hline Male & 3 & 5 & & \\
\hline \multicolumn{5}{|l|}{ Race/Ethnicity } \\
\hline Caucasian & 21 & 22 & & \\
\hline African-American & 3 & 4 & & \\
\hline Hispanic & 1 & 0 & & \\
\hline Asian/Pacific Islander & 3 & 1 & & \\
\hline \multirow[t]{2}{*}{ Other } & 2 & 3 & & \\
\hline & M & SD & M & SD \\
\hline Age & 19.5 & 1.96 & 19.6 & 1.4 \\
\hline BDI-II & 7.77 & 5.33 & 7.5 & 5.28 \\
\hline DS-R Total Score (0-100) & $65.10^{\mathrm{a}}$ & 14.71 & $47.33^{b}$ & 12.64 \\
\hline DS-R:Animal Reminder & $3.09^{a}$ & 0.57 & $1.99^{\mathrm{b}}$ & 0.75 \\
\hline DS-R: Core & $2.67^{a}$ & 0.64 & $2.11^{\mathrm{b}}$ & 0.59 \\
\hline DS-R: Contamination & 1.67 & 0.91 & 1.21 & 0.75 \\
\hline
\end{tabular}

Table 1: Differences emerged between BIl phobic and non-phobic individuals. Note. $\mathrm{BII}=$ blood-injection-injury; $M=$ mean; $S D=$ standard deviation; BDI-I I = Beck Depression Inventory-Second Edition; different superscripts would indicate a significant difference between groups. DS-R Subscale Means $=$ the average of all items belonging to theindicated subscale (in accordance with current scoring methods of the DS-R; Haidt et al., 1994,modified by Olatunji et al. 2007). Different superscripts indicate a significant difference between groups.

\begin{tabular}{|l|l|l|l|l|}
\hline & \multicolumn{3}{|c|}{ Mean D Scores } & \multicolumn{2}{l|}{} \\
\hline & Bll phobic & Non-phobic \\
\hline & M & SD & M & SD \\
\hline Pre-exposure & $0.59^{\mathrm{a}}$ & 0.34 & 0.64 & 0.32 \\
\hline Post-exposure & 0.45 & 0.34 & 0.56 & 0.3 \\
\hline Follow-up & $0.30^{\mathrm{b}}$ & 0.39 & 0.43 & 0.49 \\
\hline
\end{tabular}

Table 2: Difference in $\mathrm{D}$ scores between pre- and post-exposure.

Note. $M=$ mean; $S D=$ standard deviation. $D$ scores range between -2 and +2 ; more positive Dscores indicate a close relationship between disgust and mutilation; more negative $\mathrm{D}$ scores indicate a close relationship between appealing and mutilation; $\mathrm{D}$ scores near zero indicate no difference in association between disgust-mutilation and appealing-mutilation. Different superscripts indicate a significant difference within groups

willing to touch a spot on the table next to the deer leg with no glove on, and $70 \%$ completed the BAT in its entirety.

Hypothesis 3: Finally, it was predicted that BII phobic participants would be willing to engage in more steps of the BAT with the vial of blood after exposure to the severed deer leg, demonstrating that exposure to the severed deer leg would generalize to another animal reminder disgust-eliciting stimulus (vial of blood). This hypothesis was supported, as there was a significant difference in the number of steps that BII phobic participants engaged in from pre-exposure to followup, $\mathrm{t}(29)=-3.03, \mathrm{p}=.105$. However, no significant differences were found from pre-exposure to post-exposure, $t(29)=-1.79, p=.085$ (Table 3 ). There was no significant difference in the mean number of BAT steps that non-phobic individuals were willing to complete between pre and post-exposure, $t(29)=-1.80, p=.08$.
Independent samples t-tests were conducted to compare mean number of steps completed on the three BATs (pre-exposure, postexposure, and follow-up) and during exposure to the severed deer leg between BII phobic and non-phobic participants. At each exposure BII phobics completed significantly fewer steps than NP's (all $p$ 's $<.01$ ).

\section{Discussion}

The results of the current study indicate that a weakening of maladaptive disgust associations and decrease in avoidance behavior can be achieved in BII phobia through a relatively short treatment session (30 minutes) consisting of only in vivo disgust exposure. While BII phobic individuals did not demonstrate a significant change in $\mathrm{D}$ scores immediately following exposure, results showed a significant slowing of response times from pre-exposure to follow-up. This indicates a weakening of disgust associations and supports the first hypothesis. These results are similar to [6] work with spider phobics.

A similar pattern was found with avoidance behavior. We found a significant decrease in avoidance across time for phobics, but not nonphobics. This difference, however, only appeared in the comparison of pre- to 1-week follow-up. A potential explanation for there not being a change seen at post-exposure may have to do with the smaller sample size. The sample size constraints may have impacted our ability to see a change at post-exposure.

The second hypothesis predicted that the further a BII phobic participant progressed through the severed deer leg exposure hierarchy, the greater the difference scores in D scores $(\Delta D)$ the participant would exhibit. This hypothesis was not supported.

It should also be noted that exposure to the severed deer leg generalized to another animal reminder disgust-eliciting stimulus (vial of blood), as there was a significant difference in the number of BAT steps that BII phobic participants engaged in from pre- to postexposure. Potential implications for treatment would suggest that BII individuals might not need to be exposed to the exact feared stimulus in order for treatment to be effective. More specifically, the exact fear may be only one of the various possible stimuli utilized in treatment.

There are several limitations to the current study including sample size, sample composition, and the debrief. The smaller sample size may have served as a contributing factor in there not being significant changes at follow-up. Additionally, since the sample was comprised mainly of undergraduate students at American University, whom selfselected into this study, the generalizability of the findings are unclear. While the BII sample consisted of those meeting diagnostic criteria

\begin{tabular}{|l|l|l|l|l|}
\hline & \multicolumn{4}{|c|}{$\begin{array}{l}\text { Behavioral Approach/Avoidance Task } \\
\text { (BAT) Results }\end{array}$} \\
\hline & BII phobic & Non-phobic & \\
\hline & M & SD & M & SD \\
\hline $\begin{array}{l}\text { Pre-exposure BAT (vial } \\
\text { of blood) }\end{array}$ & $2.17^{\mathrm{a} 1}$ & 1.8 & $4.87^{\mathrm{a} 2}$ & 0.35 \\
\hline $\begin{array}{l}\text { 30-minute exposure (deer } \\
\text { leg) }\end{array}$ & $2.20^{1}$ & 2.14 & $4.53^{2}$ & 0.82 \\
\hline $\begin{array}{l}\text { Post-exposure BAT (vial } \\
\text { of blood) }\end{array}$ & $2.63^{1}$ & 2.03 & $4.97^{2}$ & 0.18 \\
\hline $\begin{array}{l}\text { Follow-up BAT (vial of } \\
\text { blood) }\end{array}$ & $3.07^{\mathrm{b} 1}$ & 2.17 & $5.00^{\mathrm{b} 2}$ & 0 \\
\hline
\end{tabular}

Table 3: Difference in the mean number of BAT.Note. $M=$ mean; $S D=$ standard deviation; means and standard deviations represent the average number of steps (0-5) completed. Different superscripts signify significant differences at $p<.05$ level; numbers indicate differences between groups and letters indicate differences within groups 
for specific phobia, it is possible that those severe phobics self-selected out of this study. Also, due to the stringent cut-offs for BII diagnostic criteria, individuals on the cusp of meeting criteria were categorized as non-phobic. Lastly, the fact that participants were debriefed at the conclusion of their initial visit may serve as another limitation in this study. It is possible that some of the improvements that we saw at follow-up may have been due to the fact that they were informed that the blood was not real.

\section{Conclusions}

Future studies should consider adding a cognitive component to strictly exposure-based intervention studies [6] and compare outcomes to cognitiveonly and exposure-only interventions to determine whether or not targeting cognitions improves outcomes in terms of behavioral avoidance and implicit associations. Studies could examine the addition of cognitive restructuring during exposure therapy to determine whether or not this addition provides incremental value in creating cognitive changes as measured by an IAT. Future studies may also want to take a dimensional approach to evaluating BII phobia, as it may offer additional insight into the phobia itself as well as changes seen through the utilization of exposure therapy.

Using the IAT as a diagnostic tool may be helpful for clinicians in the future to measure the extent to which their clients are experiencing cognitive changes. If this is the case, clinicians might consider using the IAT as a means of assessing an individual's cognitive network and maladaptive beliefs in relation to their specific phobia throughout the course of treatment.

\section{References}

1. American Psychiatric Association (2000) DSM-IV-TR. American Psychiatric Association: Arlington, VA.

2. Kleinknecht RA, Lenz J (1989) Blood/injury fear, fainting and avoidance of medically-related situations: A family correspondence study. Behaviour Research and Therapy 27: 537-547.

3. BeckAT, Clark DA (1997) An information processing model of anxiety: Automatic and strategic processes. Behaviour Research and Therapy 35: 49-58.

4. Salkovskis P M, Rachman S (1997) Frontier of Cognitive Therapy: The State of The Art and Beyond Guilford Press.

5. Teachman BA, Gregg AP, Woody SR (2001) Implicit associations for fear relevant stimuli among individuals with snake and spider fears. Journal of Abnormal Psychology 110: 226-235.

6. Teachman BA, Woody SR (2003) Automatic processing in spider phobia: implicit fear associations over the course of treatment. Journal of Abnormal Psychology 112: 100-109.

7. Olatunji BO, Connolly KM, David B (2008) Behavioral avoidance and selfreported fainting symptoms in blood/injury individuals: An experimental test of disgust domain specificity. Journal of Anxiety Disorders 22: 837-848.

8. Olatunji BO, Williams NL, Tolin DF, Sawchuck CN, Abramowitz JS, et al. (2007) The disgust scale: Item analysis, factor structure, and suggestions for refinement. Psychological Assessment 19: 281-297.

9. Tolin D F (1999) The role of disgust in blood-injection-injury phobia. The Behavior Therapist 22: 96-99.

10. Olatunji BO, Haidt J, McKay D, Bieke D (2008) Core, animal reminder, and contamination disgust: three kinds of disgust with distinct personality behavioral, physiological, and clinical correlates. Journal of Research in Personality 42: 1243-1259.

11. De Jong PJ, Merckelbach H (1998) Blood-injection-injury phobia and fear of spiders: Domain specific individual differences in disgust sensitivity. Personality and Individual Differences 24:153-158.

12. Gerlach AL, Nat R, Spellmeyer G, Vogele C, Huster R, et al. (2006) Blood-injury phobia with and without a history of fainting: Disgust sensitivity does not explain the fainting response. Psychosomatic Medicine 68: 331-339.

13. Olatunji BO, Lohr JM, Sawchuk C N, Patten K (2007) Fear and disgust responding to heterogeneous blood-injection-injury stimuli: Distinctions from anxiety symptoms. Journal of Psychopathology and Behavioral Assessment 29: 1-8.

14. Koch MD, O'Neill HK, Sawchuk CN, Connolly K (2002) Domain-specific and generalized disgust sensitivity in blood-injection-injury phobia: the application of behavioral approach/avoidance tasks. Anxiety Disorders 16: 511- 527.

15. Rusch N (2011) The role of disgust in blood-injection-injury phobia examined through the use of implicit association tests. (Master's thesis).

16. Hirai M, Cochran HM, Meyer J S, Butcher J L, Vernon LL, et al. (2008) A preliminary investigation of the efficacy of disgust exposure techniques in a subclinical population with blood and injection fears. Behaviour Change 25 : 129-148.

17. Brown TA, DiNardo PA, Barlow DH (1994) Manual for the Anxiety Disorders Interview Schedule for DSM-IV - Adult Version. New York: Oxford University Press.

18. Haidt J, McCauley C, Rozin P (1994) Individual differences in sensitivity to disgust: A scale sampling seven domains of disgust elicitors. Personality and Individual Differences 16: 701-713.

19. Beck AT, Steer R A, Brown GK (1996) Manual for the Beck Depression Inventory-II. San Antonio, TX: Psychological Corporation.

20. Storch EA, Roberti JW, Roth DA (2004) Factor structure, concurrent validity, mand internal consistency of the beck depression inventory-second edition in a sample of college students. Depression and Anxiety 19: 187-189.

21. Greenwald AG, McGhee DE, Schwartz JLK (1998) Measuring individual differences in implicit cognition: the implicit association test. Journal of Personality and Social Psychology 74: 1464-1480.

22. Connolly KM, O'Neill HK, Flessner CA, Olatunji BO (2006) Fear, disgust and fainting in blood-injection-injury fears: A multi-method approach. Anxiety, Stress, and Coping 19: 409-420.

23. Lang PJ, Bradley MM, Cuthbert BN (2008) International affective picture system (IAPS): Affective ratings of pictures and instruction manual. Technical Report A-8. University of Florida, Gainesville, FL.

24. Greenwald AG, Nosek BA, Banaji MR (2003) Understanding and using the Implicit Association Test: An improved scoring algorithm. Journal of Personality and Social Psychology 85: 197-216.

25. Smits JA, Telch MJ, Randall PK (2002) An examination of the decline in fear and disgust during exposure-based treatment. Behaviour Research and Therapy 40: 1243-1253.

26. Cisler J M, Olautnji BO, Lohr JM (2009) Disgust sensitivity and emotion regulation potentiate the effect of disgust propensity on spider fear, bloodinjection-injury fear, and contamination fear. Journal of Behavior Therapy and Experimental Psychiatry 40: 49-58.

27. Van Overveld M, de Jong PJ, Peters M L, Schouten E (2011) The Disgust Scale-R: a valid and reliable index to investigate separate disgust domains? Personality and Individual Differences 51: 325-330. 